\title{
Exploring effectiveness of simulation-based inquiry learning in science with integration of formative assessment
}

\author{
Niwat Srisawasdi ${ }^{1} \cdot$ Patcharin Panjaburee $^{2}$
}

Received: 5 January 2015/Revised: 16 June 2015/ Accepted: 18 June 2015/

Published online: 5 July 2015

(C) Beijing Normal University 2015

\begin{abstract}
This paper presents the effect of simulation-based inquiry (SimIn) integrated with formative assessment (FA) on students' conceptual learning of buoyancy-driven phenomena. In this study, we examined students' conceptual learning performance from two Thai public schools collected in two studies. In order to broaden the scope of the study's method, a two-step experiment was conducted: the first study recruited 120 ninth-grade students who agreed to participate, and they were assigned into two experimental groups and one control group. The control group received only SimIn, without integration of FA; the first experimental group participated with SimIn integrated with FA method of agree and disagree statements (SimIn-FA ${ }^{\mathrm{A} \& \mathrm{D}}$ ); and the second experimental group participated with SimIn integrated with FA method of agreement circle (SimIn-FA ${ }^{\mathrm{AC}}$ ), involving group participation. A multiple-group pretest-posttest experimental design was used, and data were analyzed using one-way ANCOVA, plus a protocol analysis was done to verify the value of the FA integration. The second study compared 39 twelfth-grade students' conceptual understanding scores, and investigated the pattern of, and the quantitative changes in, the process of conceptual change based on a one-group, pretest-posttest method. The result showed that students' conceptual understanding scores were significantly improved after participating in the experimental learning. Moreover, the integration of FA into SimIn produced a better progression of scientific understanding than without FA. This finding suggests that the integration of FA into SimIn can be used to effectively
\end{abstract}

Niwat Srisawasdi niwsri@kku.ac.th

1 Research in Science and Technology Education Group, Faculty of Education, Khon Kaen University, 123 Moo 16, Mittraphap Road, Muang, Khon Kaen 40002, Thailand

2 Institute for Innovative Learning, Mahidol University, 999, Phuttamonthon 4 Road, Salaya, Nakorn Pathom 73170, Thailand 
support construction of comprehensive understanding of science concepts in the natural setting of school science learning.

Keywords Computer simulation - Inquiry learning - Assessment of learn · Conceptual understanding $\cdot$ Conceptual change

\section{Introduction}

In constructivist views of learning among current science education researchers, students enter science classrooms with prior knowledge or conceptions about the physical world, before they ever receive formal instruction. In general, teachers encounter a variety of students' conceptual information which often is incomplete and contrary to accepted scientific knowledge, or at variance with scientifically accepted norms, formally called misconceptions or alternative conceptions, mostly based on their prior knowledge (Driver and Oldham 1986; Westbrook and Marek 1991; Vosniadou 1999). In addition, these non-scientific or alternative conceptions have been demonstrated to be pervasive and stable in the students' conceptual framework of knowledge. Researchers found that the alternative conceptions can be resistant to change through traditional classroom instruction or unstrategic teaching practices (Hewson and Hewson 1983; Nussbaum and Novak 1982; Wandersee et al. 1994). As such, there is a call for action in which students' alternative conceptions were changed, transformed, or reconstructed into the intended scientific conceptions for science-based education.

To be able to successfully restructure their concepts in science, students need to link newly constructed scientific ideas to their pre-existing concepts, refining or dismissing them in the case of incommensurability. Researchers in science education have concentrated on investigating theories and ways to facilitate the understanding of scientific concepts and bring about conceptual change (She 2004). Due to attributes of science-based contents and scientific conceptual systems by their nature, (e.g., ontological attributes and mental state, abstraction of mechanism, complexity of situation, unobservable quality of phenomena, and particular knowledge representations of communication), learning environments are required, which adhere to constructivist processes of knowledge acquisition that allow and promote students to discover new principles and explanations of the topic at hand. To achieve that, learning activity should support the active cognitive engagement of individuals rather than a process of direct transmission.

Concurrent with the rapid growth of computers and technologies in the practice of, and progressive developments in, the science education community, contemporary technology-based approaches to science learning offer computer simulations with ample opportunities for students' inquiry-related learning environments for conceptual change (de Jong and van Joolingen 1998; Rutten et al. 2012; Srisawasdi and Kroothkeaw 2014; Vreman-de Olde et al. 2013). Computer simulations are programs that contain a representation of an authentic system or phenomenon and they have a number of features that are of particular help in the teaching of science (Blake and Scanlon 2007; Wellington 2004). Currently, inquiry-based learning with computer simulations is generally seen as a promising area for conceptual change in science 
(Smetana and Bell 2012; Srisawasdi and Kroothkeaw 2014; Srisawasdi and Sornkhatha 2014). Computer simulations offer many attributes that are potentially useful for promoting cognitive dissonance and inducing conceptual change (Rutten et al. 2012). This argument is confirmed in works by Bell and Trundle (2008), and Trundle and Bell (2010), who sought to describe the impact of integrating computer simulations with inquiry-based instruction to promote conceptual change. To address the learning problems in science outlined in the previous section, simulation-based inquiry (SimIn) has increasingly become a pedagogical approach for enhancing students' conceptual learning and development in school science (Srisawasdi and Kroothkeaw 2014; Srisawasdi and Sornkhatha 2014) where they can perform their own investigations, while at the same time develop knowledge of the concept modeled by the simulation (de Jong et al. 2013; Lazonder et al. 2010; Suits and Srisawasdi 2013; Vreman-de Olde et al. 2013). Previous studies have shown that the SimIn learning environment, as an active agent in the process of conceptual change, effectively promotes better conceptual understanding in science for students (Hsu 2008; Jaakkola and Nurmi 2008; Jaakkola et al. 2011; Lazonder and Ehrenhard 2014; McElhaney and Linn 2011; Olympiou and Zacharia 2012; Olympiou et al. 2013; Renken and Nunez 2013; Srisawasdi and Kroothkeaw 2014; Srisawasdi and Sornkhatha 2014; Suits and Srisawasdi 2013; Trundle and Bell 2010; Zacharias et al. 2008). Further, in the interest of conceptual change research, it is a challenge to extend strategic ways to use SimIn for making conceptual change occur efficiently.

In classroom practice, formative assessment (FA), i.e., assessment used to inform subsequent learning, is recommended by educational researchers as an instructional strategy, which can have a considerable positive impact on student learning (Black et al. 2002; Falk 2011). Black and Wiliam (1998) reviewed 250 articles and chapters that investigated the effects of efforts to strengthen formative assessment on student achievement in comparison with controls. They provided substantial evidence of this impact and reported that it is an effective educational intervention for improve student learning. With respect to assessment for learning, formative assessment involves gathering, interpreting, and acting on information about students' learning (Bell and Cowie 2001; Duschl 2003) so that it could be used to supply valuable information for inquiry-based teaching practice in order to induce a cognitive process of conceptual change. As a classroom practice, Yin et al. (2014) embedded formal formative assessment with qualitative feedback within a curricular sequence, and they found that students' scientific understanding improved and they were promoted to change their existing conceptions. Although the impact of formative assessment is well documented and recommended by educators, little is known about the role of formative assessment on conceptual change (Shavelson et al. 2008; Yin et al. 2014). Only a few studies have been conducted to examine its influence on promoting the change of unscientific conceptions held by students. Yin et al. (2008) mentioned that simply embedding formative assessments in a curriculum did not impact students' motivation, achievement, or change their conceptual understanding. This result provided evidence for the difficulty and complexity of effectively implementing formative assessment within science learning. In addition, Yin et al. (2014) conducted a further study to obtain a better understanding of more effective ways of a formative assessment approach for 
promoting students' learning achievement and conceptual change. They shortened the formative assessment, which was used in the previous study, by half for providing timely information to help teachers and students improve teaching and learning of science. A set of formal formative assessments aligned with the learning progression of conceptual understanding were used throughout the instruction and the results showed that the formative assessment can provide multiple cognitive conflicts and challenge students' misconceptions, thus reinforcing scientific conceptions. However, Yin et al. (2014) suggested that the results only demonstrated an effect for formative assessment within a domain-specific content, and the effectiveness of the formative assessment needs to be examined with other content or concepts heavy in misconceptions. That is, we need more evidence on how to integrate formative assessment into science learning and better explanations about what happens in students' cognitive development of scientific understanding by the use of formative assessment as an integral part of pedagogy.

Although simulation-based studies have mentioned that it is an effective teaching method for conceptual development in science as well as formative assessment strategy, and hypothesized it would have beneficial impact on conceptual change, only a few studies focused on how to integrate formative assessment and SimIn to enhance conceptual learning in science. For examples, Kowalski and Kowalski (2013) implemented interactive simulation combined with real-time formative assessment in a junior level electromagnetic engineering physics course, and they reported that students were indeed more curious than they appeared to be in class, and students participated more fully even without extrinsic motivation. Chiu and Linn (2014) investigated the impact of a visualization-enhanced inquiry unit that was implemented in Web-based Inquiry Science Environment (WISE). They integrated formative assessment as a pedagogical tool for knowledge integration, and they found an advantage of the inquiry unit in consolidating students' understanding regarding connections among molecular, observable, and symbolic representations of chemical reactions. Besides, Quellmalz et al. (2012) employed science simulation as a tool for computer-assisted formative assessment in an inquiry investigation, and they found that this method can permit assessment of inquiry-based knowledge and standards in science learning. Based on our review of the literature, we found that no study has, at yet, particularly investigated the effect of formative assessment strategy into a context of SimIn learning process for students' conceptual development and conceptual change. We believed it is important to discover an emerging effective conceptual change strategy that may enhance students' learning of science concepts.

\section{Theoretical background}

\section{Simulations}

Computers have become increasingly important among educators, and play important roles in the classroom and laboratory science instruction. One type of computer application in science-based education is simulations (or computer 
simulations) and we turned our attention to examine the effects of the simulations in school science. Simulations are useful in concept learning for visualization, because of the complex and invisible nature of concepts (Rutten et al. 2012). Moreover, these gains are realized by presenting dynamic theoretical or simplified models of real-world components, phenomena, or processes, and encouraging students to observe, explore, recreate, and receive immediate feedback about real objects, phenomena, and processes (Srisawasdi and Kroothkeaw 2014). Based on visual-aid learning with simulations, the visualizations facilitate the integration cognitively of new knowledge with existing knowledge, which are important components of learning from the constructivist perspective, and improve conceptual understanding of scientific phenomena (Cook 2006; Wu and Shah 2004). In addition, simulations allow students to change variable values and observe effects to form scientific conclusions. Through this process, students discover principles, rules, and characteristics of scientific phenomena (Veemans et al. 2006), implying that they could change their conception when alternative scientific concepts exist. Therefore, computer interactive simulations can be used in enhancement of the students' conceptual change based on principles of constructivist learning theory (Dega et al. 2013).

\section{Simulation-based inquiry learning in science}

Simulation-based learning environments are considered as appropriate learning tools for manifesting conditions of conceptual change in science learning (Chen et al. 2013). Otherwise, the effectiveness of computer simulations is closely connected to the pedagogy through which they are employed (Flick and Bell 2000). In consideration of the nature of science content, integration of technology and appropriate instructional strategy is an important emphasis in recent reforms in science education. In the community of science educators, teaching and learning through scientific inquiry is recognized as an instructional practice, or learning process, which is concerned about the cognitive development of the learner and constructivist ideas of the nature of science (Hofstein and Lunetta 2004; Hofstein and Mamlok-Naaman 2007). Inquiry-based learning with simulations is a promising area for science-based instruction to foster learners' mental interaction with the physical and social world in order to develop scientific understanding, explanation, and communication among science ideas. Researchers have found that SimIn learning works as a remedial process by producing change in the alternative conceptions held by learners (Bell and Trundle 2008; Jimoyiannis and Komis 2001; Muller et al. 2008; Srisawasdi et al. 2013; Srisawasdi and Kroothkeaw 2014; Srisawasdi and Sornkhatha 2014; Windschitl and Andre 1998; Zacharia and Anderson 2003), improving the performance of gaining intuitive domain-specific knowledge, and promoting more qualitative knowledge than formalized knowledge (de Jong et al. 1999; Veemans et al. 2006; Suits and Srisawasdi 2013), achieving a more theoretical focus and coherent understanding of the concepts (Winberg and Berg 2007), and promoting positive perception of science learning (Buyai and Srisawasdi 2014; Pinatuwong and Srisawasdi 2014). 


\section{Formative assessment for science learning}

The science education research community has recently devoted considerable effort to develop how students come to learn science concepts and inquiry practices comprehensively. In order to promote such deeper learning of science, educators recognized that traditional assessment formats cannot adequately assess these aspects of science (Quellmalz et al. 2012). Currently science teachers need to understand the process by which they establish learning goals, determine what students currently know, and then to take action in the form of providing feedback to help students advance in their learning (Black and Wiliam 2009; National Research Council 2001). Educational researchers recommended formative assessment, assessment used to inform subsequent learning, as an instructional strategy, which can have a considerable positive impact on student learning (Black et al. 2002; Falk 2011). Formative assessment can take a number of forms in science classrooms. Cowie and Bell (1999) suggested two forms of planned formative assessment: (1) a teacher plans an assessment for the purpose of obtaining information about students' learning from the whole class, and (2) interactive formative assessment, in which a teacher simply pays attention and responds to student thinking during regular classroom activities, to enhance teacher-student interaction during science instruction. In addition, formative assessment could be extensively used extending from informal, on-the-fly assessment, which is often carried out in conversation with students and when teachers are checking in on groupwork, to formal, curriculum-embedded activities (Shavelson et al. 2008). In a previous study, formative assessment was used during the learning process to provide feedback for students and teachers in order to improve both learning and teaching. Zuiker and Whitaker (2014) studied how formative assessment could support flexible inquiry-based learning, and they found that multiple-level assessment made student thinking visible and pedagogical decision-making more powerful in science classes. In addition, Yin et al. (2014) examined the effect of learning progression-aligned formal embedded formative assessment on conceptual change and achievement in an inquiry-based curriculum for middle-school science, and they indicated that embedding formal formative assessments within a curricular sequence provides multiple cognitive conflicts to challenge students' misconceptions and reinforce scientific conceptions, by promoting conceptual change. Accordingly, given its documented benefits in science learning, formative assessment, therefore, should be considered to be used as a valuable source of information in inquiry-based teaching practice in science classrooms.

\section{Enhancing science learning through conceptual change}

In recent years, to promote students' scientific understanding and conceptual change, several researchers have attempted to develop instructional materials and research approaches for enhancing conceptual learning in science. The findings from the research studies showed the importance of a cognitive domain on learners' process of conceptual change toward better conceptual understanding in science (e.g., She 2004; She and Liao 2010; Srisawasdi et al. 2013; Srisawasdi and 
Kroothkeaw 2014). However, how conceptual change happens or how students progress during conceptual understanding in science is a somewhat blurry picture for many science educational researchers. Many researchers have proposed theories that explain the difficulties encountered by students when learning certain scientific concepts, and the how the cognitive processes functioned when the students transformed an existing framework of conceptions. Posner et al. (1982) and Strike and Posner (1985) explained how conceptual change happens in science based on Piaget's cognitive development theory. In their theory, they pointed out that conceptual change is oriented to a cognitive process of accommodation, which refers to large-scale conceptual changes, in contrast to assimilation, which refers to learning in which a major conceptual revision is not required (She 2004). In addition, Thagard (1992) proposed a spectrum of possible degrees of conceptual change, from changes in relatively surface-level details to radical shifts in core concepts, by considering a treelike structure of conceptual framework as follows: (a) adding a new instance; (b) adding a weak rule; (c) adding a new strong rule; (d) adding a new-part relation; (e) adding a new-kind of relation; (f) adding a new concept; (g) collapsing part of a particular hierarchy; (h) branch jumping; and (i) tree switching. In an effort to further explain cognitive processes of conceptual change during students' learning in science, Dykstra et al. (1992) introduced a taxonomy to characterize the unique way that conceptual representation changes from pre-conception to post-conception and the unique features of the strategies which seem to induce cognitive process of conceptual change as follows: (a) differentiation, (b) class extension, and (c) reconceptualization. Through this taxonomy, the students' cognitive processes of conceptual change were captured. Moreover, in an attempt to quantify the mechanical change of students' conceptual understanding, She and Liao (2010) proposed the idea for analysis of conceptual change as follows: (a) progress (PG); (b) maintain-correct (MTC); (c) maintainpartial correct (MTPC); (d) maintain-incorrect (MTIC); (e) retrogression (RTG). Researchers argue that actively engaging students in the process of conceptual change are important for development of conceptual understanding in science. Careful examination of the process involved in the conceptual change reveals how students progressed from prior knowledge or preexisting conceptions toward a more scientific view of the concepts. Therefore, the above-mentioned theoretical ideas could be used to describe whether students change their existing conceptual understanding into another form or pattern of understanding both during and after interacting with any instructional intervention or learning environment.

\section{Study context}

In our study, we recognized the importance of a pipeline of evidence that begins with a quantitative well-designed controlled trial, as a primary evidence for evaluating an intervention's effectiveness, and then ends with a qualitative study. The purpose of this research is to discover whether the benefit of formative assessment strategy in a SimIn learning environment for students' conceptual learning performance can contribute to the accumulation of empirical evidence and development of theoretical models. We have conducted two distinct studies in 
which we have used SimIn integrated with FA, where the practice of formative assessment is used by teachers during a SimIn learning process to provide feedback for both teachers and students. Moreover, they can act upon the feedback to improve teaching and learning, to structure students' conceptual development of buoyancydriven phenomena. In the first study, we explored the effect of the SimIn integrated with FA in relatively controlled classroom contexts (Plass et al. 2009), and this study was conducted in the complexity of everyday classroom practice (O'Donnell 2008). Such study of effectiveness is designed to address whether an intervention "does more good than harm when delivered under real-world conditions" (Plass et al. 2012). In addition, Means and Penuel (2005) noted that investigations of technology-based innovations should be scaled up to address the question, "What works, when and how?". As such, the second study has been conducted to provide phenomenological evidence of the intervention on how it works in developing students' conceptual learning performance. We believed this approach would be helpful by locating our study in a more thorough design, and hopefully getting a more robust finding.

In order to improve effectiveness of SimIn, the science formative assessment proposed by Keeley (2008) was considered and the strategy of "Agree and Disagree (A\&D) Statement" and "Agreement Circle (AC)" were particularly selected as a part of the intervention. In this study, the $A \& D$ statement is a formative assessment idea in which students use a series of flag cards to respond to each conceptual statement individually, identifying their agreement, disagreement, or neither agree or disagree. For the idea of agreement circle, students use a couple of flag cards to respond to a series of conceptual questions individually for representing their existing understanding. Both strategies provide opportunity to activate thinking and engage learners to describe and discuss their own ideas with peers and teacher. As they engage in these strategies, learners immediately received formative feedback, which can provide useful information for correcting misconceptions (Shute 2008). According to its features, these strategies could be rapidly implemented to help the teacher make students' ideas explicitly evident and conveniently support teacher collecting information about what students know and do not know in an interactive way. In addition, A\&D statements provide an opportunity for students to practice metacognition as well as during the Agreement Circle strategy (Keeley 2008). These formative assessment strategies were used as an integral part, at the concluding step, of the simulation-based open-inquiry learning process (Srisawasdi and Kroothkeaw 2014; Srisawasdi and Sornkhatha 2014). Through these formative assessment strategies, a set of two to four statements or questions, relating to the concept being studied in computer simulation, was presented to the students after they had experienced the experimental learning treatment to determine how they would flag the process of their learning as a form of feedback.

\section{The Buoyancy simulation by PhET}

To promote development of conceptual understanding on why things float and sink, an interactive computer simulation on buoyancy obtained from Physics Education Technology (PhET) research group at University of Colorado, Boulder, was used as 


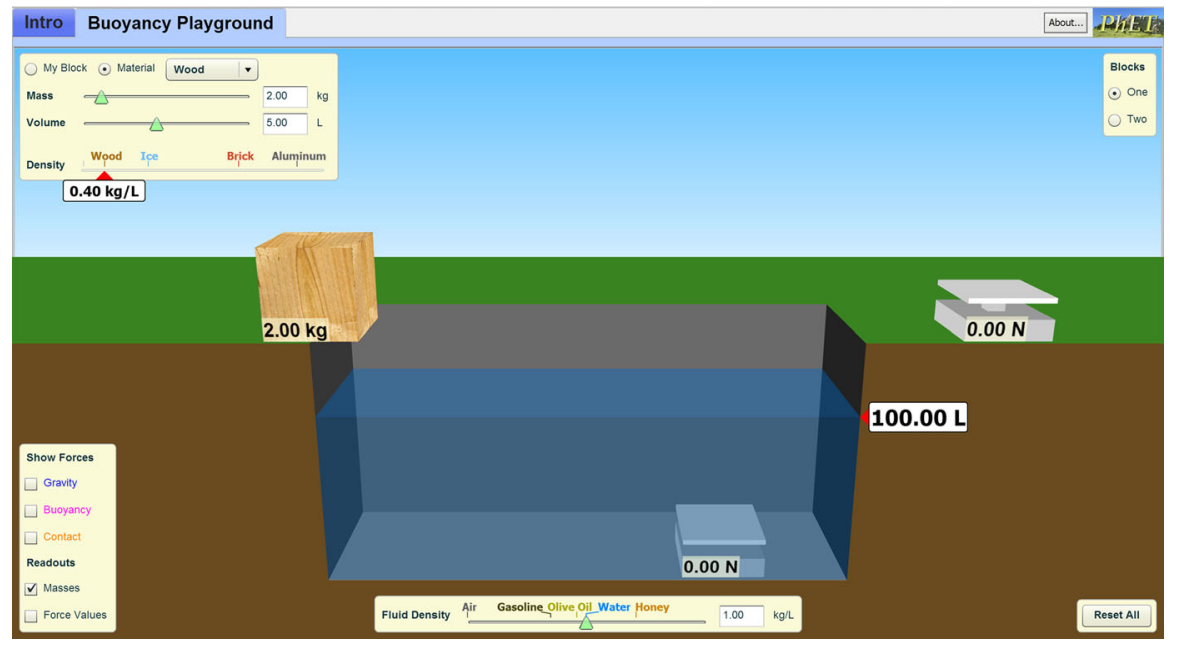

Fig. 1 An illustrative interface example of the buoyancy simulation from PhET

inquiry tool for students. It is clear that students' common alternative conceptions of buoyancy are due to the invisibility of amount involved and their physical nature, making it more difficult to construct concepts related to buoyancy. Therefore, the buoyancy simulation was designed and developed to address the common alternative conceptions held by students at all levels, and the design emphasizes providing students with visualizations of the buoyancy-driven phenomena to help them build more scientific views of buoyancy concepts. For example, one part of the buoyancy simulation allows students interacting with it to understand the introduction to buoyancy, wherein students learn how buoyancy works using blocks made of different materials. Arrows show the applied forces, and students can modify the properties of the blocks and the fluid as displayed in Fig. 1.

\section{Study 1: the effect of formative assessment on conceptual learning for simulation-based inquiry}

The goal of this study was to investigate students' conceptual understanding delivered including formative assessment that was integrated into the SimIn context. According to the research goal, two questions were explored in this study. First, does formative assessment strategy affect students' conceptual learning achievement in a SimIn learning context? Second, what kind of formative assessment strategy best suits the SimIn learning context? Results from the first study are intended to indicate the instructional value that formative assessment adds into SimIn in a science learning context. Moreover, findings from the first study inform the second study in order to investigate effectiveness of the method on students' cognitive performance of conceptual development. 


\section{Research design}

In our effort to investigate the effect of SimIn with and without formative assessment, a multiple-group pretest-posttest experimental design procedure was selectively employed in order to answer the research questions, comprising two independent variables namely SimIn integrated with FA strategy (with three levels: SimIn-FA ${ }^{\text {A\&D }}$, SimIn-FA ${ }^{\text {AC }}$, and SimIn used as a control), as shown in Fig. 2.

To provide more details, the SimIn integrated with FA method of agree and disagree statements (SimIn-FA ${ }^{\mathrm{A} \& D}$ ) refers to teaching practice by stimulating curiosity and interest through an open-ended inquiry question, and then providing essential theory and basic scientific background to foster testable hypotheses generation, supported by using computer simulation for both steps. Afterward, students were assigned to design their own experiment and perform the experiment with the simulation, and then analyze obtained data to verify the visualized phenomena. Next, the teacher organized a class communication by presenting the results and then a discussion of the main idea ensued. After finishing the presentation, students were presented with a series of conceptual statements and they were allowed to response to each statement individually by using flag cards, identifying their agreement, disagreement, or neither agree or disagree. Moreover, some of them were asked, as representatives of the answer, to explain the reason why they agree or disagree with the statement, and then discuss together with peers. In the end, the teacher provided formative feedback on the statements by using the simulation and then assisted them to draw a conclusion based on evidence obtained

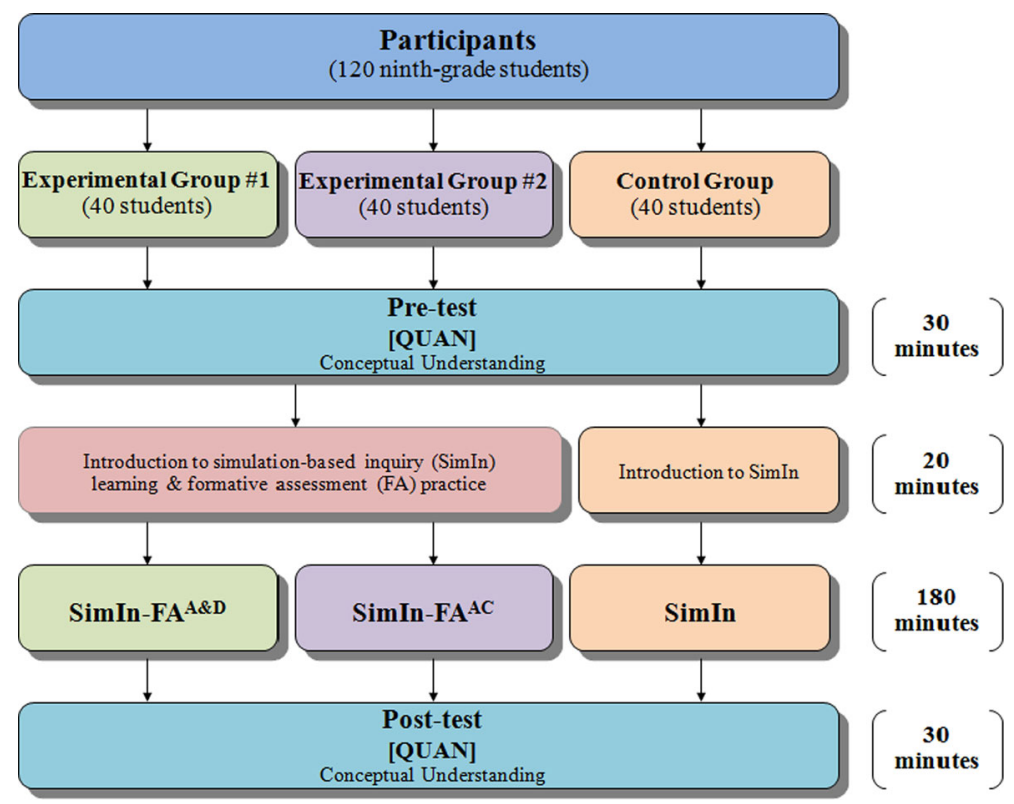

Fig. 2 Experimental procedure of this study 
from the simulation in order to modify their thinking as new information replaces, or becomes assimilated into, their existing conceptual knowledge. In the case of the SimIn integrated with FA method of agreement circle $\left(\operatorname{SimIn-FA}{ }^{\mathrm{AC}}\right.$ ), this assessment strategy provided a kinesthetic way to respond to the provided statements rather than the use of flag card only. After identifying their agreement or disagreement, the teacher encouraged the students who agree with the statement to step into the center of the circle. Students who agree and disagree with the statement turn to face their peers and then some of them were selected to explain the reason defending their own thinking. Next, they were allowed to reposition themselves with those who now agree standing in the center of the circle. This situation was repeated with several rounds of the statements before moving to the conclusion step.

\section{Study participants}

A total of 120 student-respondents in their ninth-grade, aged ranging from 14 to 15 years old in a local public school at Northeast region of Thailand participated in this study. They came from three classes. Two classes were assigned to be the experimental groups and one the control group. The first experimental group (EG\#1, $N=40$ ) interacted with the SimIn integrated with FA method of agree and disagree statement (SimIn-FA ${ }^{\mathrm{A} \& D}$ ), and the second experimental group (EG\#2, $N=40$ ) participated with SimIn integrated with FA method of agreement circle (SimIn$\mathrm{FA}^{\mathrm{AC}}$ ), and the control group (CG, $N=40$ ) was exposed to SimIn only. They were attending a science course for basic education level and all groups were taught by the same teacher. Based on an informal interview with the regular class instructor before starting the experimental study, the result indicated that all of them had satisfactory skills for using basic computer and information and communication technology, but they had no experience yet using a computer simulation in science learning. In addition, they had never experienced a formal class with SimIn. This implied that they were heterogeneous in background before interacting with the simulations in the experimental study.

\section{Measures and data analysis}

A series of two-tier conceptual questions was produced based on a summary of common misconceptions about why things sink and float reported by Yin et al. (2008). The researcher translated the list and adapted it into ten two-tier question items in Thai language. The items were multiple-choice and open-ended type in the first and second tier, respectively. For the test, items must be answered correctly in both content and reason tiers to be scored as one point, and the maximum score is ten points. Examples of the two-tier question items are presented in Appendix 1. The question items were content validated by a physicist and a physics teacher. The focus of the question items was different from those asked in the formative assessment sessions, which emphasized conceptual information that was presented in the computer simulation only, but the science concepts were similar and more open-ended. All items assessed conceptual understanding of relative density, determined by floating and sinking. The internal reliability coefficient as calculated 

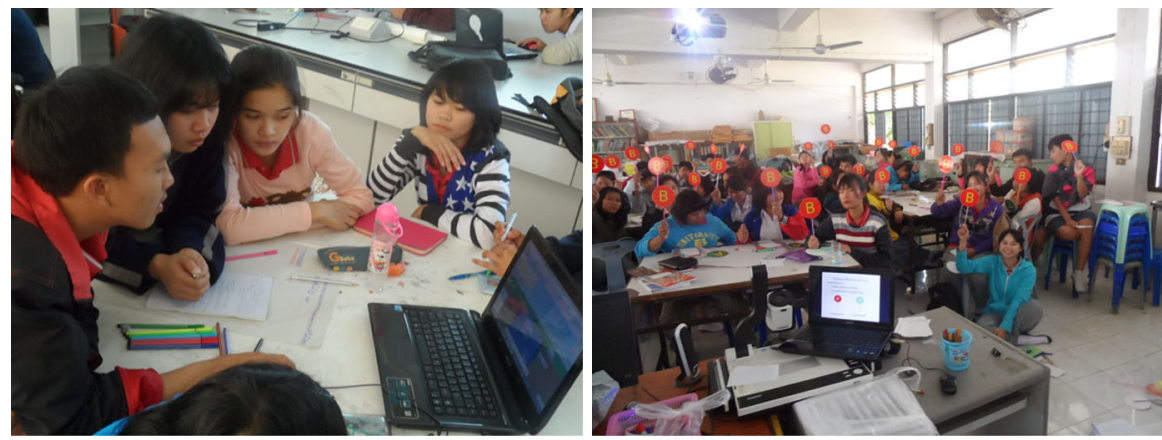

Fig. 3 Illustrative examples of learning activity through simulation-based inquiry integrated with formative assessment: inquiry learning with simulation (left) and formative assessment practice (right) showing the students displaying cards

by Kuder-Richardson Formula 20 (KR20) was .74. All three groups were examined for conceptual understanding by using the ten two-tier question items for $30 \mathrm{~min}$, both pretest and posttest. They were also given an orientation to the learning activity and familiarized with the simulation and formative assessment during 20 min after the pretest. The experimental groups experienced the SimIn integrated with FA and the control group learned with the SimIn for three two-hour weekly classes. During the learning activity of SimIn as presented in Fig. 1, the experimental groups received content-specific formative assessment practice twice during a learning activity. The teacher enacted formative assessment practices with the following goals: (i) to measure and support students' learning progress with simulation, and (ii) to elicit and respond to students' understanding and thinking. All groups experienced four conceptual learning events on floating and sinking phenomena including: (i) density, (ii) buoyancy, (iii) floating, and (iv) sinking, in this study, as illustrated in Fig. 3.

For answering the research questions, one-way analysis of covariance (ANCOVA) was used to compare the scores of the three groups in terms of conceptual understanding among the experimental and control groups. Paired t-tests were also used to examine difference in the conceptual understanding scores for each group, before and after instructional intervention.

\section{Research findings}

\section{Analysis of conceptual understanding score}

The one-way analysis of variance (one-way ANOVA) was done between the conceptual understanding scores of the experimental groups and the control group on the pretest administered before the teaching experiment. For the pretest measure of conceptual understanding, the EG\#1 group had a mean of 2.15 (SD =1.54); the EG\#2 group had a mean of $.90(\mathrm{SD}=1.08)$; and the $\mathrm{CG}$ group had a mean of 1.95 $(\mathrm{SD}=1.76)$. Analysis indicated that there was a statistically significant difference 
Table 1 ANCOVA results for the experimental groups and the control group on conceptual understanding scores

\begin{tabular}{llrlllll}
\hline Source & $\begin{array}{l}\text { Type III sum } \\
\text { of squares }\end{array}$ & $d f$ & $\begin{array}{l}\text { Mean } \\
\text { squares }\end{array}$ & $F$ & Sig. & $\begin{array}{l}\text { Partial } \\
\eta^{2}\end{array}$ & $\begin{array}{r}\text { Observed } \\
\text { power (a) }\end{array}$ \\
\hline Group & 334.988 & 2 & $\begin{array}{r}167.494 \\
1.695\end{array}$ & 98.834 & $.000 * * *$ & .630 & 1.000 \\
Error & 196.586 & 116 & & & & \\
\hline
\end{tabular}

$R^{2}=.630$ (adjusted $R^{2}=.621$ )

$* p<.05 ; * * p<.01 ; * * * p<.001$

between the experimental groups and the control group on the pretest score $(F(2,117)=8.09, p=.001)$. This implies that the three groups had different levels of conceptual understanding on floating and sinking phenomena before the experimental teaching. To reduce the threats of sample error, the one-way ANCOVA was utilized to test the main effects for the experiment groups and the control group on conceptual understanding, controlling the effects of prior conception. The dependent variable, covariates, and independent variable were posttest measurement of conceptual understanding, pretest measurement of conceptual understanding, and teaching condition, respectively. Before the ANCOVA was done, the homogeneity test within groups regression coefficient was executed. The result showed that the F value was equal to $.25(p=.251)$. This suggests that the homogeneity test has not reached statistical significance. It means that there is no significant difference in variances among the three groups. Therefore, the ANCOVA could be done. Table 1 reports the result of the ANCOVA analysis.

The result of the ANCOVA indicates that there is a statistically significant difference $(F(2,116)=98.834, p=.000)$ between the experimental groups and the control group after the intervention as shown in Table 1. After eliminating the influence of covariance (pretest), the EG\#1 group had an adjusted mean of 6.47 $(\mathrm{SE}=.21)$; the EG\#2 group had an adjusted mean of $6.11(\mathrm{SE}=.22)$, and the $\mathrm{CG}$ group had an adjusted mean of $2.74(\mathrm{SD}=.21)$ for the posttest measure of conceptual understanding. Students who participated in the SimIn-FA A\&D group outperformed others; and students in the SimIn-FA ${ }^{\mathrm{AC}}$ group were better than the SimIn group. In other words, after the instruction (integrating formative assessment versus not integrating formative assessment), the students in the experimental groups had enhanced their conceptual understanding on floating and sinking phenomena. In addition, a closer look at the $r^{2}=.630$ implies that the intervention accounted for $63.0 \%$ of the total variation in the learning effect of the experimental treatment. This indicates that the SimIn, integrated with FA into the science learning of floating and sinking phenomena, has a significant impact on conceptual understanding and is therefore superior to a more general SimIn learning.

To determine where the differences among the teaching methods were, Bonfferoni's Post Hoc Test was employed to test for significance. All tests were conducted using the adjusted means, controlling for any differences in prior conception. Table 2 reports the result of the Bonfferoni's pairwise comparisons. 
Table 2 Bonferroni Post Hoc Test results by teaching condition

\begin{tabular}{|c|c|c|c|}
\hline (I) Group & (J) Group & Mean difference (I-J) & Sig. \\
\hline \multirow[t]{2}{*}{ CG (SimIn) } & EG\#1 (SimIn-FA A\&D) & -3.727 & $.000 * * *$ \\
\hline & EG\#2 (SimIn-FA $\left.{ }^{\mathrm{AC}}\right)$ & -3.369 & $.000 * * *$ \\
\hline \multirow[t]{2}{*}{ EG\#1 (SimIn-FA $\left.{ }^{\text {A\&D }}\right)$} & CG (SimIn) & 3.727 & $.000 * * *$ \\
\hline & EG\#2 (SimIn-FA $\left.{ }^{\mathrm{AC}}\right)$ & .358 & .741 \\
\hline \multirow[t]{2}{*}{ EG\#2 (SimIn-FA $\left.{ }^{\mathrm{AC}}\right)$} & CG (SimIn) & 3.369 & $.000 * * *$ \\
\hline & EG\#1 (SimIn-FA A\&D) & -.358 & .741 \\
\hline
\end{tabular}

$* p<.05 ; * * p<.01 ; * * * p<.001$

Table 3 Descriptive statistics and paired t-test on the pretest and posttest for the experimental groups and the control group

\begin{tabular}{|c|c|c|c|c|}
\hline Group & $\begin{array}{l}\text { Pretest } \\
\text { Mean (SD) }\end{array}$ & $\begin{array}{l}\text { Posttest } \\
\text { Mean (SD) }\end{array}$ & $t$ & $p$ \\
\hline CG (SimIn) & $1.95(1.76)$ & $2.78(1.36)$ & 2.860 & $.007 * *$ \\
\hline EG\#1 (SimIn-FA $\left.{ }^{\text {A\&D }}\right)$ & $2.15(1.54)$ & $6.53(1.39)$ & 13.688 & $.000 * * *$ \\
\hline EG\#2 (SimIn-FA $\left.{ }^{\mathrm{AC}}\right)$ & $.90(1.08)$ & $6.03(1.14)$ & 19.095 & $.000 * * *$ \\
\hline
\end{tabular}

$* p<.05 ; * * p<.01 ; * * * p<.001$

The post hoc test for the teaching condition variable was tested at the preestablished alpha level of .05. The CG group were compared to the EG\#1 and EG\#2 groups revealing a mean difference of 3.727, $p=.000$, and of $3.369, p=.000$, respectively, indicating a significant difference between the SimIn integrated with FA (SimIn-FA ${ }^{\mathrm{A} \& \mathrm{D}}$ and SimIn-FA ${ }^{\mathrm{AC}}$ ) and the group without formative assessment (SimIn). This evidence indicated that integration of formative assessment caused better effects on helping students establish scientific conceptions. This finding is consistent with Zuiker and Whitaker (2014), i.e., that the enactment of formative assessment could complement the inquiry-based learning process in order to develop collective conceptual understanding of the scientific phenomena. In addition, the comparison of the EG\#1 group with the EG\#2 revealed a mean difference of .358 and level of significance of $p=.741$ that indicated that there was no significant difference between the two teaching methods of SimIn-FA ${ }^{A \& D}$ and SimIn-FA ${ }^{\mathrm{AC}}$ as is depicted in the data reported in Table 2.

In addition, Table 3 reports the results of the paired $t$ test between pretest and posttest for all groups. The result of the paired $t$ test reveals that there is a statistically significant difference between pretest and posttest for the EG\#1 group $(t=13.68, p=.000)$, EG\#2 group $(t=19.09, p<.001)$, and CG group $(t=2.86$, $p=.007)$. The results highlight the benefits of using formative assessment along with SimIn learning activities to promote students' conceptual understanding of floating and sinking phenomena. These results suggest that the formative assessment strategy can help students to better understand the theoretical principles of buoyancy-driven physical phenomena under the context of SimIn learning. In overall conclusion, these results suggest that the FA integrated into SimIn is more 
beneficial for students' conceptual learning performance than the SimIn without integration of FA.

\section{Study 2: a difference in conceptual learning and cognitive performance in simulation-based inquiry integrated with formative assessment}

As we have shown, there is a learning value of the SimIn integrated with FA, which enhanced students' conceptual understanding in science. In this second study, we investigate a phenomenon of conceptual development in science to answer the following specific questions: (1) Can students' conceptual change be promoted using SimIn integrated with FA? and (2) What are students' cognitive mechanisms of conceptual change that occurred in context of SimIn integrated with FA? Results from the second study could be to broaden perspectives and yield a more comprehensive understanding about impacts of SimIn integrated with FA.

\section{Research design}

In our effort to investigate the efficacy of SimIn integrated with FA using an agreement circle (SimIn-FA ${ }^{\mathrm{AC}}$ ), we used a combination of quantitative and qualitative research methodologies in this study. The concurrent embedded strategy of the mixed research methodology was conducted by embedding a qualitative research methodology within a quantitative research methodology. For this study, more specifically, a phenomenological research methodology was embedded into a one-group pretest-posttest quasi-experimental design, as shown in Fig. 4.

\section{Study participants}

The participants for this study included 39 twelfth-grade students in a local public school at a Northeast region of Thailand. They were attending a physics course at a basic education level and they were invited to participate in this research. The participants were 18 years old. All of them did have satisfactory basic computer and information and communication technology skills, but they had no prior experience with using the computer in physics learning.

\section{Domain of conceptual learning events}

To facilitate the students' conceptual understanding of buoyancy-driven phenomena in this study, there were seven of the designed learning events of buoyancy that were used to cover students' alternative conceptions of the concepts consisting of: physical quantity involved when an object appears in liquid (C1); the force exerted when the object appears in liquid (C2); the relationship between the density of the liquid with the buoyancy (C3); the relationship between density and buoyancy of an object (C4); the relationship between the shape of an object with buoyancy (C5); the relationship between mass and the depth of an object with buoyancy (C6); and the effect of buoyancy on two different object (C7). 


\section{Measures and data analysis}

For investigating students' conceptual abilities, a psychological state that has the content of the rule or principle that guides and explains the concept's correct application, was examined in this study. A series of seven open-ended conceptual questions with the abovementioned conceptual attributes was created by the researchers, and validated by two physics teachers and a physicist. For the test, the score of each item ranged from 0 to 3 points, and the rubric scores were as follows: 0 point, demonstrates no attempt, or no scientific understanding (full of alternative conceptual understanding); 1 point, demonstrates the combination of scientific understanding (both full and limited conceptual understanding) and alternative fragment of conceptual understanding, plus any significant error of understanding; 2 points, demonstrates some scientific understanding, a limited or incomplete conceptual understanding, with no alternative fragment of conceptual understanding; and 3 points, demonstrates complete scientific understanding (full of conceptual understanding). The rubrics were constructed by, and results interpreted through consensus of, the two researchers and the two physics teachers; thus ensuring that the rubrics were properly evaluated and relevant to the specific concepts of buoyancy-driven phenomena. As such, this numerical score represented students' level of conceptual understanding about floating and sinking, and the maximum score was 21 for this test. Examples of the open-ended question items are presented in Appendix 2. The open-ended question items were administered to examine the students' conceptual understanding before they attended the SimIn-FA ${ }^{\text {AC }} 45-\mathrm{min}$ teaching method. The SimIn-FA ${ }^{A C}$ method was presented during 4, 3-h weekly classroom sessions, and included seven conceptual learning events on floating and sinking phenomena. Figure 5 illustrates examples of the SimIn integrated with FA with agreement circle (SimIn-FA ${ }^{\mathrm{AC}}$ ), showing students participating in agreement circles.

Finally, a 45-min posttest was administered after the experiments were completed. For the posttest, the same open-ended conceptual question items were

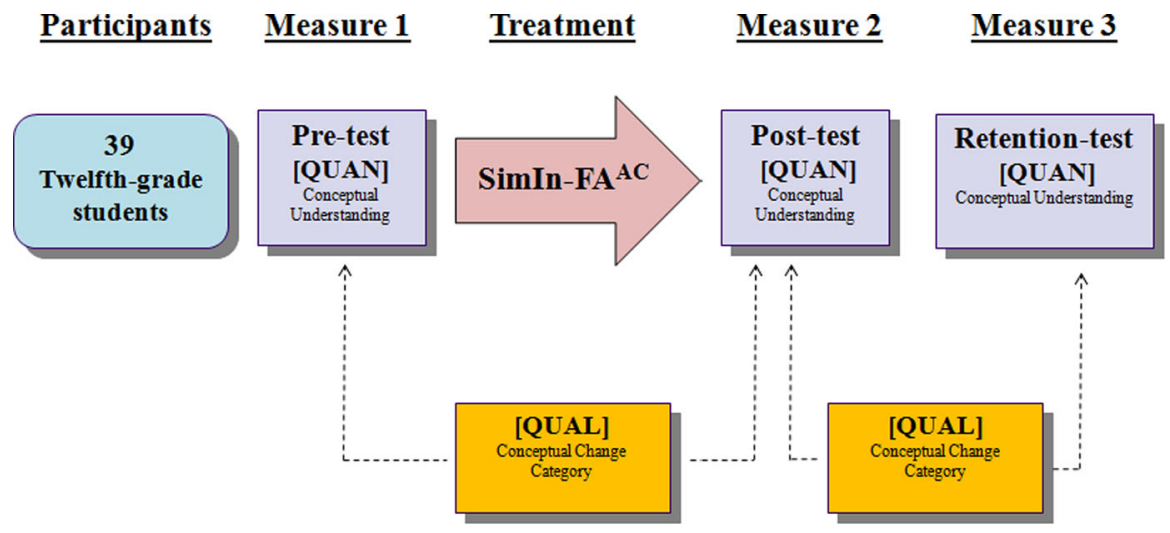

Fig. 4 Structure of the research design used in the study 

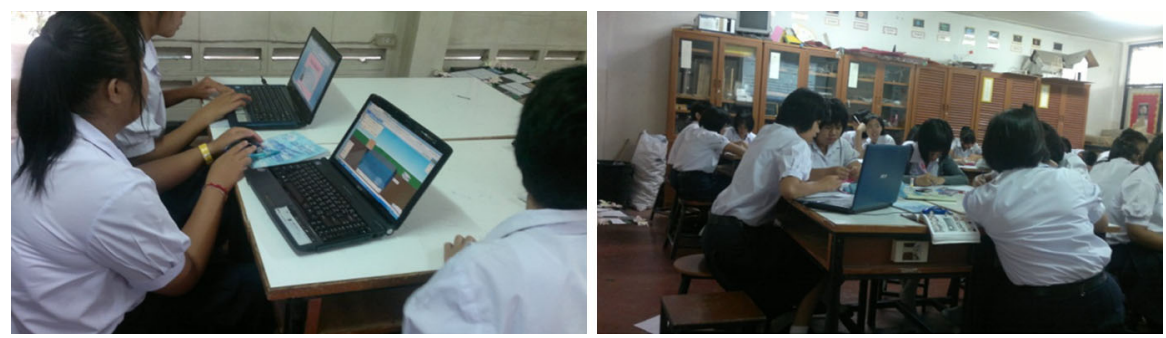

Fig. 5 Illustrative examples of learning activity through SimIn-FA ${ }^{\mathrm{AC}}$

administered to the students again to assess the status of their current conceptual understanding, and to investigate conceptual change. To analyze the students' conceptual understanding, content analysis was primarily used to write a protocol of their answers to each open-ended question item from both pretest and posttest. Then, the rubric was used to evaluate the conceptual quality of their understanding. Because the data obtained from the rubric scores were not normally distributed, a nonparametric Friedman's Chi-square test and Wilcoxon sign-ranked test were used to statistically determine differences for their conceptual understanding scores. Moreover, for students' conceptual change analysis, the qualitative changes of their conceptual understanding between pretest to posttest, were analyzed and assigned into five categories (She and Liao 2010) including: (1) Progress (PG) - to what extent the student's conceptions improved; (2) MTC - to what extent the student's conceptions were maintained correctly: (3) MTPC - to what extent the student's conceptions were maintained as partially correct; (4) MTIC - to what extent the student's conceptions were maintained as partially incorrect; (5) Retrogression (RTG) - to what extent the student's conceptions retrogressed. Each student's conceptual understanding results were analyzed as percentages for PG, MTC, MTPC, MTIC, and RTG from pretest to posttest, and then posttest to retention-test. To qualitatively examine the students' cognitive processes of conceptual change during the students' interactions with the SimIn integrated with FA, the classroom verbal protocols, and the transcripts of video and audio records, were analyzed by discourse analysis. The technique of discourse analysis is the study of the vital link between text and the broader social context of its production and consists of three interrelated processes of analysis: (1) description of the text; (2) interpretation of the relationship between the text and the social processes of the interaction; and (3) explanation of the relationship between the social processes and the social context (Fairclough 2003).

The researchers began by transcribing the video and audio recordings and repeatedly reading the transcripts while identifying and labeling the linguistic features in the protocol. Then, the role of the researchers is to interpret the cognitive process of conceptual change, as manifested in the protocols, based on a taxonomy of conceptual change (Dykstra et al. 1992): (a) Differentiation-wherein new concepts emerge from existing, more general concepts; (b) Class extensionwherein existing concepts considered different are found to be cases of one 
subsuming concept; and (c) Reconceptualization-wherein a significant change in the nature of and relationship between concepts occurs. The categories in this taxonomy are characterized by unique changes in conceptual representation from pre-conception to post-conception and by unique features of the strategies which seem to induce the type of conceptual change represented by those categories (Dykstra et al. 1992).

\section{Research findings}

\section{Analysis of conceptual understanding score}

In order to explore the effect of the SimIn-FA ${ }^{\mathrm{AC}}$ on the students' conceptual understanding of floating and sinking phenomena, Table 4 presents results of the statistical comparative analysis for students' pretest, posttest, and retention-test scores using nonparametric Friedman's Chi-square test and Wilcoxon sign-ranked test.

The results shows a significantly difference among pretest, posttest, and retention-test mean scores. In addition, it indicated that the students made substantial gains in their conceptual understanding of buoyancy from pretest to posttest as indicated by the posttest score being significantly greater than the pretest $\left(Z=-5.456, p_{\text {(post }>\text { pre })}<.001\right)$. Also, evidence of major shift in their conceptual understanding was found based on a difference between pretest and retention-test mean scores. The retention-test score was significantly greater than the pretest $\left(Z=-5.456, p_{\text {(post }>\text { pre })}<.001\right)$. Furthermore, they also made slight gains from posttest to retention test and the difference was also significantly $(Z=-3.690$, $\left.p_{\text {(post }>\text { pre })}<.001\right)$.

\section{Analysis of conceptual change category}

The percentages for the amount of conceptual change from pretest to posttest are presented in Fig. 6. As seen in Fig. 6 for pretest to posttest, the percentage of the Progress (PG) category was higher than any other category across most of the seven concepts, except for the last concept (C7), where MTIC was the highest. The percentage for Progress (PG) ranged from 35.90 to 100.00 , MTC category ranged from 2.56 to 10.26; there were no occurrences of the 5th concept (C5); MTIC was found only on the 3rd (C3) and 7th (C7) concept categories ranging from 43.59 to

Table 4 Statistical results of Friedman's Chi-square test and Wilcoxon sign-ranked test analysis on conceptual understanding scores

\begin{tabular}{lrrrll}
\hline Measurement & Mean & Median & S.D. & Asymp. Sig. & Pairwise comparison \\
\hline Pretest & 2.72 & 3.00 & 1.50 & $.000^{*}$ & Posttest $>$ Pretest* \\
Posttest & 15.21 & 15.00 & 2.15 & & Posttest $>$ Retention-test* \\
Retention-test & 16.15 & 16.00 & 2.58 & & Retention $>$ Pretest* \\
\hline
\end{tabular}

$* p<.05$ 


\section{(A)}

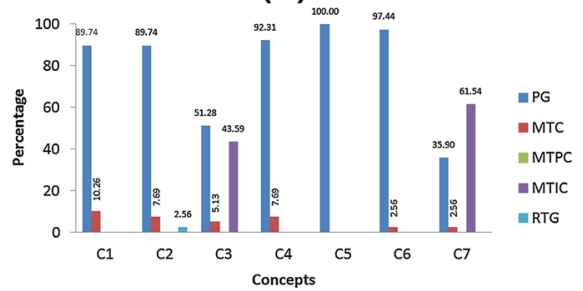

(B)

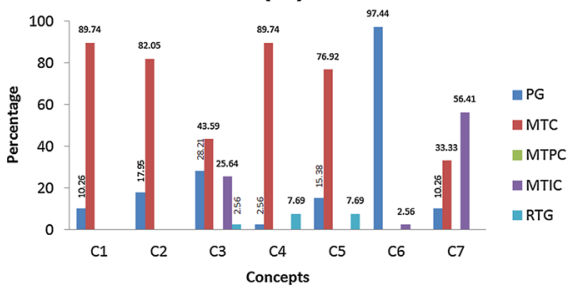

(C)

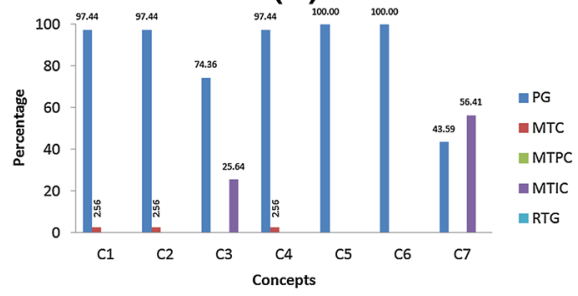

Fig. 6 Distribution of conceptual change across seven concepts-related buoyancy-driven physical phenomena; a pretest to posttest; b posttest to retention-test; c pretest to retention-test

61.54, and Retrogress (RTG) was 2.56 on the 2nd concept (C2). MTPC was not found for any concepts.

Figure 6 displays the percentage of the quantity of conceptual change from posttest to retention-test across seven concepts of the buoyancy phenomena, including the percentage of change for students' understanding of buoyancy from posttest to retention-test. The percentage of MTC category was higher than other categories across most of the seven concepts, except for the last concept (C7), where MTIC was the highest. The progress (PG) category evidence was found on the 1st, 2nd, 3rd, 4th, and 7th concepts and the percentages for PG ranged from 2.56 to 28.21. The MTIC category was found on the 3rd and 7th concepts, and the percentages for both concepts were 25.64 and 56.41, respectively. The retrogress (RTG) category percentages were 2.56 for the 3rd and 6th concepts and 7.69 for the 4 th and 5th concepts. The MTPC category was not found for any of the concepts.

\section{Analysis of the cognitive mechanism of conceptual change}

Conceptual change is a complex cognitive process that need intervention, and cannot be effected through student information sharing by itself, or by a transmission model of learning (Dykstra et al. 1992; Hake 1998). The cognitive mechanism of conceptual change is a progressive process of refinement of students' conceptions (Demastes et al. 1996; Dykstra et al. 1992; Niedderer and Goldberg 1994). In order to capture the cognitive process of conceptual change in science learning, Srisawasdi and Kroothkeaw (2014), and Srisawasdi and Sornkhatha (2014) employed Dykstra et al.'s (1992) taxonomy of conceptual change, consisting of Differentiation, Class extension, and Reconceptualization, in discourse protocol analysis of videotaped and audio-taped transcripts recorded during students' interact 


\title{
Cognitive Mechanism of Conceptual Change
}

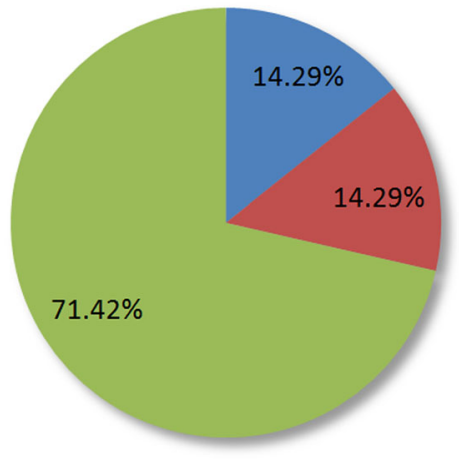

\author{
Differentiation \\ Class Extension \\ Reconceptualization
}

Fig. 7 Distribution of conceptual change mechanism during the SimIn-FA ${ }^{\mathrm{AC}}$ classes

with SimIn. They found that student conceptual understanding had been progressively improved and refined, going from alternative conceptions to some intermediate conceptions and scientific conceptions.

To examine the hypothesized merits of integration of FA into SimIn in this study, the classroom verbal protocol, the transcripts of video and audio data, were analyzed by discourse analysis. The researchers began with the transcribed video and audio recordings, and repeatedly read the transcripts while identifying and labeling the linguistic features in the protocol. By these steps, some manageable chunks of verbal protocol for illustrating the cognitive process were found for the interpretation of the classroom setting. Then, the analyst interpreted the cognitive process of conceptual change, as manifested in the protocol, based on a taxonomy of conceptual change (Dykstra et al. 1992), and we found overall evidence of cognitive conceptual change in seven protocol excerpts of data during student interaction with the SimIn-FA ${ }^{\mathrm{AC}}$. Figure 7 displays percentages of the quantity of conceptual change based on analyses using the taxonomy.

In addition, Table 5 presents results of evidence for cognitive mechanism of conceptual change obtained from students' pretest and posttest using discourse protocol analysis. Based on these data, the cognitive process of reconceptualization has the highest percentage of conceptual change mechanism, where the differentiation and class extension are equal. This indicates that most of the students seem to change the nature of buoyancy-driven concepts and the relationship between the concepts as a result of interacting with the SimIn-FA ${ }^{\mathrm{AC}}$.

\section{Discussion and conclusions}

This study evaluated the effectiveness of simulation-based inquiry integrated with FA (SimIn-FA) targeted at conceptual learning of science within Thai school science curriculum. In line with previous studies on SimIn learning in science 


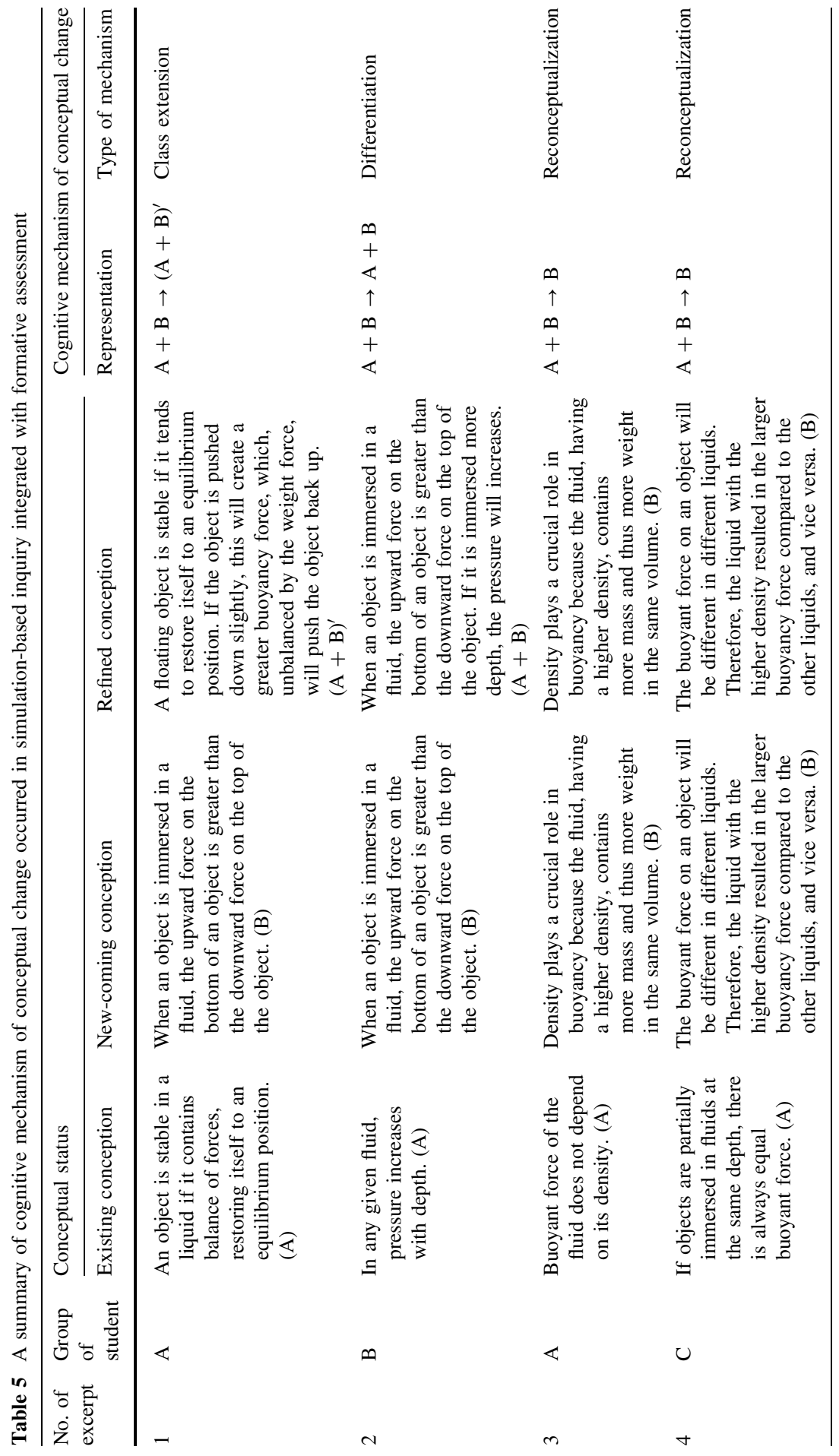




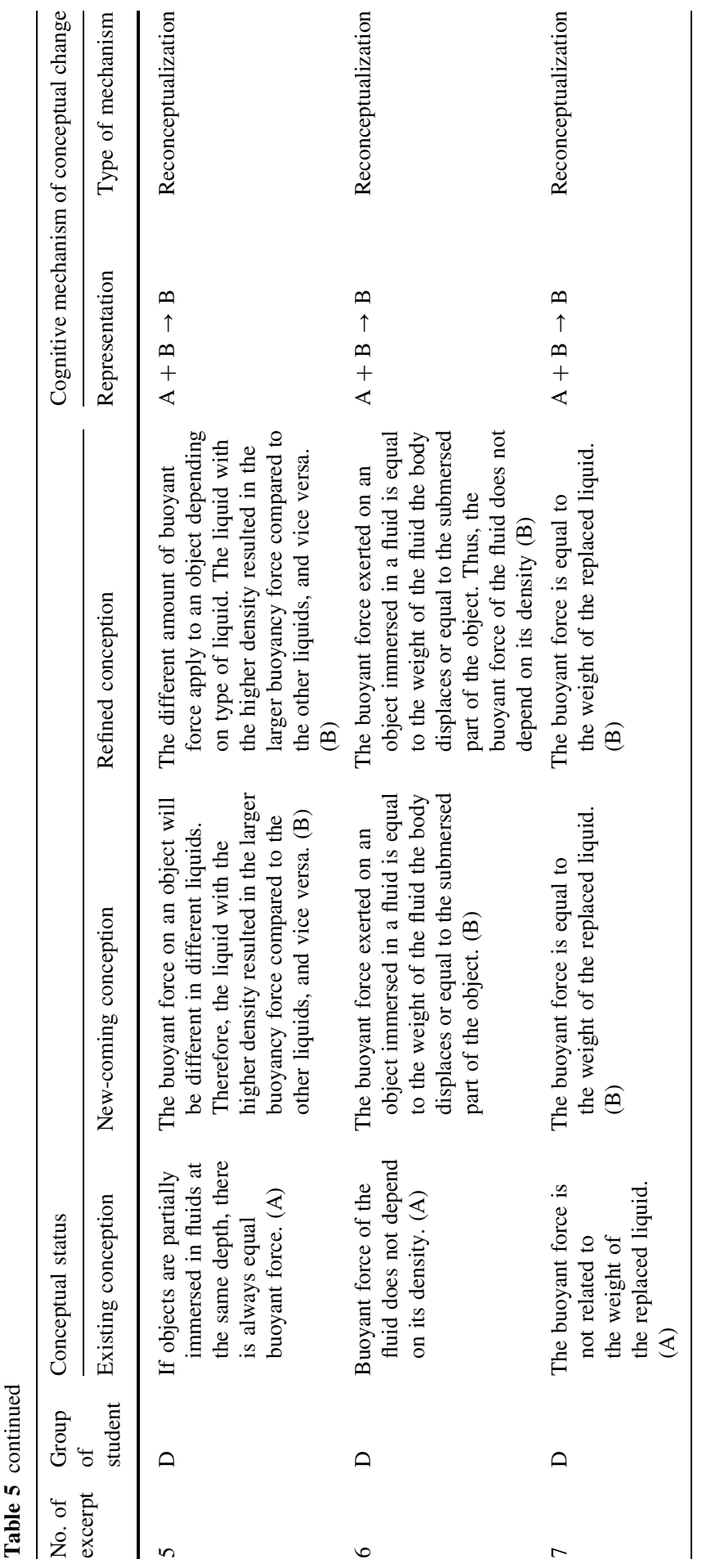


(Hsu 2008; Jaakkola and Nurmi 2008; Jaakkola et al. 2011; Lazonder and Ehrenhard 2014; McElhaney and Linn 2011; Olympiou and Zacharia 2012; Olympiou et al. 2013; Renken and Nunez 2013; Srisawasdi et al. 2013; Srisawasdi and Kroothkeaw 2014; Srisawasdi and Sornkhatha 2014; Suits and Srisawasdi 2013; Trundle and Bell 2010; Zacharias et al. 2008), the results from both of our two studies indicated that secondary school students had better conceptual achievement of science by interacting with inquiry-based learning of science by simulation. These findings indicate, and could be argued, that SimIn-FA in secondary education can be used to enhance students' conceptual development of science concepts and their understanding on buoyancy-driven concepts, in particular. Moreover, the intervention of SimIn-FA made a durable change throughout the students' conceptual learning in science. Through the SimIn-FA activity, it seems that the agreement circle strategy is the most effective, compared with the agree and disagree approach, in helping students establish scientific conceptions through SimIn learning.

In additions, findings of this study are consistent with previous studies on formative assessment practice in science instruction (Yin et al. 2014), i.e., embedding formal formative assessments can promote conceptual change in science classrooms. Results from this study provided a number of interesting qualitative findings as to why students made progress on conceptual understanding in science, and further suggested that integration of FA into SimIn could induce the progressive process of conceptual refinement through cognitive mechanism of conceptual change. The formative assessment practice could serve as a catalyst in SimIn and produced a significant conceptual change among students who hold alternative conceptions. This is apparent in the finding that the integration of formative assessments, both agreement and disagreement statements, and agreement circle, into SimIn promotes students gaining better understanding about buoyancy-driven concepts than when they only interacted with the SimIn. This argument is consistent with Yin et al. (2014) that the components of formative assessments are aligned with Posner's conceptual change model by explicitly eliciting students' thinking. Thus, they have an opportunity to evaluate their own existing conceptions, encouraging the students to take action to close the gap between prior and new conceptions. Furthermore, they can restructure their prior knowledge and work toward developing a more scientifically sound conception.

Evidence obtained by the quantitative analysis of conceptual change indicated that the students' understanding of buoyancy-driven concepts made continuous progress throughout the learning of the SimIn-FA, from pretest to posttest and posttest to retention-test. The percentage of change in the Progress (PG) category, from pretest to posttest, and the percentage of the MTC category, from posttest to retention-test, were higher than any other category across most of the seven concepts, except for the last concept (C7). This indicates that the integration of FA into SimIn successfully promoted students' conceptual change. According to the results on transitional change of conceptual understanding on the concept of buoyancy on two different objects (C7), most of the students lacked sufficient scientific conception regarding multiple variables in explaining a scientific phenomenon, even if they have interacted with the SimIn-FA. This result is consistent with $\mathrm{Wu}$ et al.'s (2013) observation that understanding about multiple 
causality involved recognition and identification of the integration rules of multiple effects and the attributes of variables. Also, Kuhn (2007) stated that students often encounter difficulties with multiple variables in science investigations. One reason might be that the conceptual learning event lacked sufficient information to provide specific mental sets the students need to develop and use to effectively foster radical conceptual change of the concept, given their cognitive level. Consequently, they did not master the skills of multiple variables in science investigations. Moreover, the formative assessment may not have created enough dissonance in the students' perceptions of evidence about buoyancy-driven concepts to bring about change. Also, their prior limited understanding about multiple causality may prevent their gaining a conceptual change. Based on this finding, the use of SimIn-FA may require additional refinements in the design of the conceptual learning events in addressing multiple causality of scientific phenomena, and thus to better use formative assessment in an appropriate and supportive manner to enhance learning of science concepts that involves multiple variables in scientific phenomena. This is also consistent with Yin et al. (2008) that simply embedding formative assessment in a lesson and curricula does not guarantee improvement of students' learning of science concepts. To promote students' conceptual change in difficult science concepts, Yin et al. (2014) suggested that the formative assessment should be embedded formally to provide multiple cognitive conflicts rather than a single cognitive conflict to challenge students' misconceptions. This design may reinforce accurate scientific conceptions and be better aligned with the learning goals of enhancing conceptual change.

In conclusion, this study provides a conceptual account of SimIn-FA method and an empirical analysis of the final enactment of a year-long effort to faithfully appropriate and adapt the method in science classrooms. Together, these two research endeavors focus on simulation-based inquiry (SimIn), formative assessment (FA), and their harmonization toward better pedagogical decision-making. In turn, we describe critical adaptations of the model that either accommodate or, at times, take advantage of local conditions. The SimIn-FA is an expanding instructional method based on earlier works (Srisawasdi et al. 2013; Srisawasdi and Kroothkeaw 2014; Srisawasdi and Sornkhatha 2014), and the present study has expanded the scope and application to include digital technology in education. It appears that this new pedagogy has the potential to provide researchers and practitioners with more prescriptive heuristics and recommendations to enhance teaching of science with SimIn lessons. The application of this pedagogy in classroom may encourage science educators and/or teachers to broaden instructional perspectives to employ more effective formative assessment methods for teaching with digital technology.

Acknowledgments This material is based on the project financially supported by the National Research Council of Thailand (NRCT) (Grant No. 2555250551884); the NRCT and Khon Kaen University (KKU) (Grant No. 570701); and the NRCT and Mahidol University (MU) (Grant Nos. 67/2557 and 103/2558). Any opinions, findings, and conclusions or recommendations expressed in this materials are of the authors and do not necessarily reflect the view of the NRCT, KKU, and MU. The authors would like to express their gratitude to Mr. Thaworn Pollayuth, a physics teacher at Thaliwittaya School, Office of The Basic Education Commission, Thailand, and Mr. Jakkrapan Sutatho, a physics teacher at Paisarnpitayakhom 
School, Office of The Basic Education Commission, Thailand, and all the secondary school students involved in this study. The authors acknowledge the commitment, time, and expert advice provided by the Guest Editors in improving this paper.

\section{Appendix 1}

1) Block A and Block B both float in water. Suppose that we glue them firmly together (see the picture below).

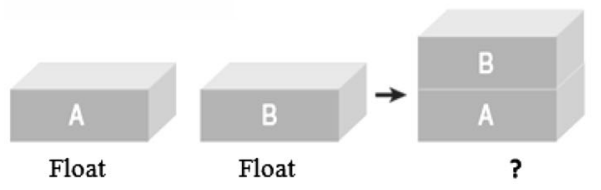

1.1) If the connected block is placed in water, what would happen?
A. They will sink
B. They will float

1.2) Why? Please describe.

2) Ball A and Ball B are made of different materials, but they have the SAME mass and the SAME volume. Ball A is solid and it sinks in water. Ball B is hollow in the center (see the pictures below).

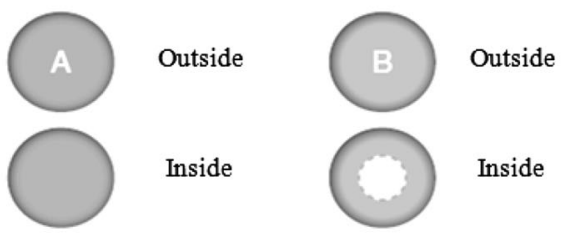

2.1) If Ball B is placed in water, what would happen?
A. They will sink
B. They will float

2.2) Why? Please describe. 


\section{Appendix 2}

1) In the below pictures, Block $A$ and Block $B$, substances with the SAME volume, are placed at the SAME depth into the identical barrels filled with DIFFERENT liquid.
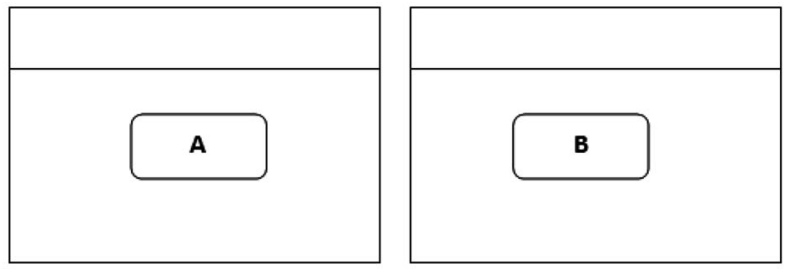

Please write your explanations below.

2) In the below pictures, Block $A$ and Block B, substances with the SAME volume, are placed at DIFFERENT level of depth into the identical barrels filled with the SAME liquid.

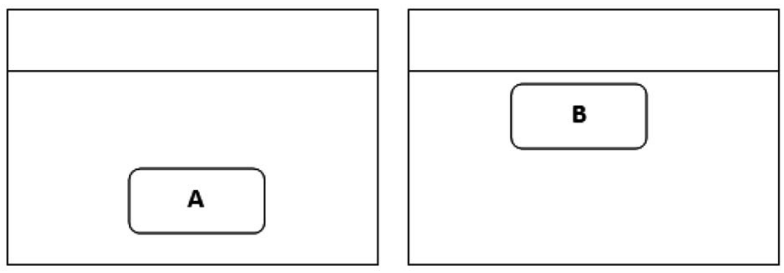

Please write your explanations below.

\section{References}

Bell, B., \& Cowie, B. (2001). The characteristics of formative assessment in science education. Science Education, 85(5), 536-553.

Bell, R. L., \& Trundle, K. C. (2008). The use of a computer simulation to promote scientific conceptions of moon phases. Journal of Research in Science Teaching, 45(3), 346-372.

Black, P., Harrison, C., Lee, C., Marshall, B., \& Wiliam, D. (2002). Working inside the black box: Assessment for learning in the classroom. London: GL Assessment.

Black, P., \& Wiliam, D. (1998). Assessment and classroom learning. Assessment in Education, 5(1), $7-74$.

Black, P., \& Wiliam, D. (2009). Developing the theory of formative assessment. Educational Assessment, Evaluation and Accountability, 21(1), 5-31.

Blake, C., \& Scanlon, E. (2007). Reconsidering simulations in science education at a distance: Features of effective use. Journal of Computer Assisted learning, 23(6), 491-502.

Buyai, J., \& Srisawasdi, N. (2014). An evaluation of macro-micro representation-based computer simulation for physics learning in liquid pressure: Results on students' perceptions and attitude. In C.-C. Liu et al. (Ed.), Proceedings of the 22nd International Conference on Computers in Education (pp. 330-339). Nara, Japan: Asia-Pacific Society for Computers in Education.

Chen, Y. L., Pan, P. R., Sung, Y. T., \& Chang, K.-E. (2013). Correcting misconceptions on electronics: Effects of a simulation-based learning environment backed by a conceptual change model. Educational Technology \& Society, 16(2), 212-227. 
Chiu, J. L., \& Linn, M. C. (2014). Supporting knowledge integration in chemistry with a visualizationenhanced inquiry unit. Journal of Science Education and Technology, 23(1), 37-58.

Cook, M. P. (2006). Visual representations in science education: The influence of prior knowledge and cognitive load theory on instructional design principles. Science Education, 90(6), 1073-1091.

Cowie, B., \& Bell, B. (1999). A model of formative assessment in science education. Assessment in Education: Principles, Policy \& Practice, 6(1), 101-116.

de Jong, T., Linn, M. C., \& Zacharia, Z. C. (2013). Physical and virtual laboratories in science and engineering education. Science, 340, 305-308.

de Jong, T., Martin, E., Zamarro, J., Esquembre, F., Swaak, J., \& van Joolingen, W. R. (1999). The integration of computer simulation and learning support: An example from the physics domain of collisions. Journal of Research in Science Teaching, 36(5), 97-615.

de Jong, T., \& van Joolingen, W. R. (1998). Scientific discovery learning with computer simulations of conceptual domains. Review of Educational Research, 68(2), 179-202.

Dega, B. G., Kriek, J., \& Mogese, T. F. (2013). Students' conceptual change in electricity and magnetism using simulations: A comparison of cognitive perturbation and cognitive conflict. Journal of Research in Science Teaching, 50(6), 677-698.

Demastes, S. S., Good, R. G., \& Peebles, P. (1996). Patterns of conceptual change in evolution. Journal of Research in Science Teaching, 33(4), 407-431.

Duschl, R. A. (2003). Assessment of inquiry. In J. M. Atkin \& J. E. Coffey (Eds.), Everyday assessment in the science classroom (pp. 41-59). Arlington, VA: National Science Teachers Association Press.

Driver, R., \& Oldham, V. (1986). A constructivist approach to curriculum development in science. Studies in Science Education, 13(1), 105-122.

Dykstra, D., Boyle, C., \& Monarch, I. (1992). Studying conceptual change in learning physics. Science Education, 76(6), 615-652.

Fairclough, N. (2003). Analysing discourse: Textual analysis for social research. London: Routledge.

Falk, A. (2011). Teachers learning from professional development in elementary science: Reciprocal relations between formative assessment and pedagogical content knowledge. Science Education, 96(2), 265-290.

Flick, L., \& Bell, R. (2000). Preparing tomorrow's science teachers to use technology: Guidelines for science educators. Contemporary Issues in Technology and Teacher Education, 1(1), 39-60.

Hake, R. R. (1998). Interactive-engagement versus traditional methods: A six-thousand-student survey of mechanics test data for introductory physics courses. American Journal of Physics, 66(1), 64-74.

Hewson, G. N., \& Hewson, W. P. (1983). Effect of instruction using student prior knowledge and conceptual change strategies on science learning. Journal of Research in Science Teaching, 20(8), $731-743$.

Hofstein, A., \& Lunetta, V. N. (2004). The laboratory in science education: Foundations for the twentyfirst century. Science Education, 88(1), 28-54.

Hofstein, A., \& Mamlok-Naaman, R. (2007). The laboratory in science education: The state of the art. Chemistry Education Research and Practice, 8(2), 105-107.

Hsu, Y. (2008). Learning about seasons in a technologically enhanced environment: The impact of teacher-guided and student-centered instructional approaches on the process of students' conceptual change. Science Education, 92(2), 320-344.

Jaakkola, T., \& Nurmi, S. (2008). Fostering elementary school students' understanding of simple electricity by combining simulation and laboratory activities. Journal of Computer Assisted learning, 24(4), 271-283.

Jaakkola, T., Nurmi, S., \& Veermans, K. (2011). A comparison of students' conceptual understanding of electric circuits in simulation only and simulation-laboratory contexts. Journal of Research in Science Teaching, 48(1), 71-93.

Jimoyiannis, A., \& Komis, V. (2001). Computer simulations in physics teaching and learning: A case study on students' understanding of trajectory motion. Computers \& Education, 36(2), 183-204.

Keeley, P. (2008). Science formative assessment-75 practical strategies for linking assessment, instruction and learning. California: NSTA press and Corwin Press.

Kowalski, F. V. \& Kowalski, S. E. (2013). Enhancing curiosity using interactive simulations combined with real-time formative assessment facilitated by open-format questions on tablet computers. Paper presented at Proceedings of Frontiers in Education Conference, Seattle, WA. Available from: http:// arxiv.org/pdf/1308.1110v1.pdf. Accessed Mar 23, 2015.

Kuhn, D. (2007). Reasoning about multiple variables: Control of variables is not the only challenge. Science Education, 91(5), 710-716. 
Lazonder, A. W., \& Ehrenhard, S. (2014). Relative effectiveness of physical and virtual manipulatives for conceptual change in science: How falling objects fall. Journal of Computer Assisted learning, 30(2), 110-120.

Lazonder, A. W., Hagemans, M. G., \& de Jong, T. (2010). Offering and discovering domain information in simulation-based inquiry learning. Learning and Instruction, 20(6), 511-520.

McElhaney, K. W., \& Linn, M. C. (2011). Investigations of a complex, realistic task: Intentional, unsystematic, and exhaustive experimenters. Journal of Research in Science Teaching, 48(7), $745-770$.

Means, B., \& Penuel, W. R. (2005). Scaling up technology-based educational innovations. In C. Dede, J. P. Honan, \& L. C. Peters (Eds.), Scaling up success: Lessons from technology-based educational improvement (pp. 176-197). San Francisco, CA: Jossey-Bass.

Muller, D. A., Sharma, M. D., \& Reimann, P. (2008). Raising cognitive load with linear multimedia to promote conceptual change. Science Education, 92(2), 278-296.

National Research Council. (2001). Knowing and learning mathematics for teaching: Proceedings of a workshop. Washington, DC: National Academy Press.

Nussbaum, J., \& Novak, S. (1982). Alternative frameworks, conceptual conflict and accommodation: Toward a principled teaching strategy. Instructional Science, 11(3), 183-200.

O'Donnell, C. (2008). Defining, conceptualizing, and measuring fidelity of implementation and its relationship to outcomes in K-12 curriculum intervention research. Review of Educational Research, $78(1), 33-84$.

Olympiou, G., \& Zacharia, Z. C. (2012). Blending physical and virtual manipulatives: An effort to improve students' conceptual understanding through science laboratory experimentation. Science Education, 96(1), 21-47.

Olympiou, G., Zacharia, Z. C., \& de Jong, T. (2013). Making the invisible visible: Enhancing students' conceptual understanding by introducing representations of abstract objects in a simulation. Instructional Science, 41(3), 575-587.

Pinatuwong, S., \& Srisawasdi, N. (2014). An investigation of relationships between biology attitudes and perceptions toward instructional technology in analogy-based simulation on light reaction. In C.-C. Liu et al. (Ed.), Proceedings of the 22nd international conference on computers in education (pp. 149-152). Nara, Japan: Asia-Pacific Society for Computers in Education

Plass, J. L., Homer, B. D., Milne, C., Jordan, T., Kaluyga, S., Kim, M., \& Lee, H. (2009). Design factors for effective science simulations: Representation of information. International Journal of Gaming and Computer-mediated Simulations, 1(1), 16-35.

Plass, J. L., Milne, C., Homer, B., Schwartz, R. N., Hayward, E., Jordan, T., et al. (2012). Investigating the effectiveness of computer simulations for chemistry learning. Journal of Research in Science Teaching, 49(3), 394-419.

Posner, G. J., Strike, K. A., Hewson, P. W., \& Gertzog, W. A. (1982). Accommodation of a scientific conception: Toward a theory of conceptual change. Science Education, 66(2), 211-227.

Quellmalz, E., Timms, M., Buckley, B., Davenport, J., Loveland, M., \& Silberglitt, M. (2012a). 21st century dynamic assessment. In M. Mayrath, J. Clarke-Midura, \& D. Robinson (Eds.), Technologybased assessments for 21st century skills: Theoretical and practical implications from modern research (pp. 55-90). Charlotte, NC: Information Age Publishers.

Quellmalz, E. S., Timms, M. J., Silberglitt, M. D., \& Buckley, B. C. (2012b). Science assessments for all: Integrating science simulations into balanced state science assessment systems. Journal of Research in Science Teaching, 49(3), 363-393.

Renken, M. D., \& Nunez, N. (2013). Computer simulations and clear observations do not guarantee conceptual understanding. Learning and Instruction, 23, 10-23.

Rutten, N., van Joolingen, W. R., \& van der Veen, J. T. (2012). The learning effects of computer simulations in science education. Computers \& Education, 58(1), 136-153.

Shavelson, R. J., Young, D. B., Ayala, C. C., Brandon, P. R., Furtak, E. M., Ruiz-Primo, M., et al. (2008). On the impact of curriculum-embedded formative assessment on learning: A collaboration between curriculum and assessment developers. Applied Measurement in Education, 21(4), 295-314.

She, H. C. (2004). Fostering "radical" conceptual change through dual-situated learning model. Journal of Research in Science Teaching, 41(2), 142-164.

She, H. C., \& Liao, Y. W. (2010). Bridging scientific reasoning and conceptual change through adaptive web-based learning. Journal of Research in Science Teaching, 47(1), 91-119.

Shute, V. J. (2008). Focus on formative feedback. Review of Educational Research, 78(1), 153-189. 
Smetana, L. K., \& Bell, R. L. (2012). Computer simulations to support science instruction and learning: A critical review of the literature. International Journal of Science Education, 34(9), 1337-1370.

Srisawasdi, N., Junphon, S., \& Panjaburee, P. (2013). Effect of simulation-based inquiry with dualsituated learning model on change of student's conception. In S. C. Tan et al. (Ed.), Proceedings of the 21st international conference on computers in education (pp. 147-154). Bali, Indonesia: AsiaPacific Society for Computers in Education

Srisawasdi, N., \& Kroothkeaw, S. (2014). Supporting students' conceptual learning and retention of light refraction concepts by simulation-based inquiry with dual-situated learning model. Journal of Computers in Education, 1(1), 49-79.

Srisawasdi, N., \& Sornkhatha, P. (2014). The effect of simulation-based inquiry on students' conceptual learning and its potential applications in mobile learning. International Journal of Mobile Learning and Organisation, 8(1), 24-49.

Strike, K. A. \& Posner, G. J. (1985). A conceptual change view of learning and understanding. In L. T. West \& A. L. Pines (Eds.), Cognitive structure and conceptual change (pp. 147-176). Orlando, FL: Academic Press.

Suits, J. P. \& Srisawasdi, N. (2013). Use of an interactive computer-simulated experiment to enhance students' mental models of hydrogen bonding phenomena. In J. P. Suits \& M. J. Sanger (Eds.) Pedagogic roles of animations and simulations in chemistry courses (pp. 241-271). ACS Symposium Series 1142, American Chemical Society: Washington, DC.

Thagard, P. (1992). Conceptual revolutions. Princeton, NJ: Princeton University Press.

Trundle, K. C., \& Bell, R. L. (2010). The use of a computer simulation to promote conceptual change: A quasi-experimental study. Computers \& Education, 54(4), 1078-1088.

Veemans, K., van Joolingen, W., \& de Jong, T. (2006). Use of heuristics to facilitate scientific discovery learning in a simulation learning environment in a physics domain. International Journal of Science Education, 28(4), 341-361.

Vosniadou, S. (1999). Conceptual change research: State of the art and future directions. In W. Schnotz, S. Vosniadou, \& M. Carretero (Eds.), New perspectives on conceptual change (pp. 1-14). Amsterdam: Pergamon.

Vreman-de Olde, C., de Jong, T., \& Gijlers, H. (2013). Learning by designing instruction in the context of simulation-based inquiry learning. Educational Technology \& Society, 16(4), 47-58.

Wandersee, J. H., Mintzes, J. J., \& Novak, J. D. (1994). Research on alternative conceptions in science. In D. L. Gabel (Ed.), Handbook of research on science teaching and learning (pp. 177-210). New York: MacMillan.

Wellington, J. (2004). Using ICT in teaching and learning science. In R. Holliman \& E. Scanlon (Eds.), Mediating science learning through information and communications technology (pp. 51-78). London: Open University Press.

Westbrook, S. L., \& Marek, E. A. (1991). A cross-age study of student understanding of the concept of diffusion. Journal of Research in Science Teaching, 28(8), 649-660.

Winberg, T. M., \& Berg, C. A. R. (2007). Students' cognitive focus during a chemistry laboratory exercise: Effects of a computer-simulated prelab. Journal of Research in Science Teaching, 44(8), 1108-1133.

Windschitl, M., \& Andre, T. (1998). Using computer simulations to enhance conceptual change: The roles of constructivist instruction and student epistemological beliefs. Journal of Research in Science Teaching, 35(2), 145-160.

Wu, H.-K., \& Shah, P. (2004). Thinking with representations: Exploring visuospatial thinking in chemistry. Science Education, 88(3), 465-492.

Wu, H.-K., Wu, P. H., Zhang, W. X., \& Hsu, Y. S. (2013). Investigating college and graduate students' multivariable reasoning in computational modeling. Science Education, 97(3), 337-366.

Yin, Y., Shavelson, R. J., Ayala, C. C., Ruiz-Primo, M., Brandon, P. R., Furtak, E., et al. (2008). On the impact of formative assessment on student motivation, achievement, and conceptual change. Applied Measurement in Education, 21(4), 335-359.

Yin, Y., Tomita, M. K., \& Shavelson, R. J. (2014). Using formal embedded formative assessments aligned with a short-term learning progression to promote conceptual change and achievement in science. International Journal of Science Education, 36(4), 531-552.

Zacharia, Z., \& Anderson, O. (2003). The effects of an interactive computer-based simulation prior to performing a laboratory inquiry-based experiment on students' conceptual understanding of physics. American Journal of Physics, 71, 618. 
Zacharias, G. L., MacMillan, J., \& Van Hemel, S. B. (2008). Behavioral modeling and simulation: From individuals to societies. Washington, D.C.: National Academies Press.

Zuiker, S. J., \& Whitaker, J. R. (2014). Refining inquiry with multi-form assessment: Formative and summative assessment functions for flexible inquiry. International Journal of Science Education, 36(6), 1037-1059.

Niwat Srisawasdi had received his Ph.D. degree in Science and Technology Education from the Institute for Innovative Learning, Mahidol University in Thailand. He is currently working as an Assistant Professor of Science Education at the Division of Science, Mathematics, and Technology Education, Faculty of Education, Khon Kaen University, Thailand. He is interested in technology-enhanced science education, and technological pedagogical and content knowledge for science teacher. He is the corresponding author of this paper.

Patcharin Panjaburee had received her Ph.D. degree in Science and Technology Education from the Institute for Innovative Learning, Mahidol University in Thailand. She is currently working as an Assistant Professor of Computer Education at the Institute for Innovative Learning, Mahidol University, Thailand. She is interested in computer-assisted testing, expert systems, knowledge engineering, technology-enhanced learning, and computer science and mathematics education. 